\title{
In Situ Observations Using Ultrahigh Vacuum Scanning Electron Microscopy
}

\author{
Y. Homma \\ NTT Basic Research Laboratories, Nippon Telegraph and Telephone Corporation, Atsugi, Kanagawa \\ 243-0198, Japan
}

Scanning electron microscopy (SEM) is a conventional observation technique, so it is not regarded as a tool for atomic layer imaging. However, SEM can image the topmost surface structures such as atomic steps [1] and reconstructed surface domains [2] in ultrahigh vacuum (UHV), just as reflection electron microscopy or low energy electron microscopy can. Since large working distances are allowed for both the primary electron beam irradiation and secondary ion detection in SEM, it is relatively easy to combine SEM with molecular beam epitaxy [3] or scanning tunneling microscopy (STM) [4]. Also, SEM can image 3D structures as well. On the other hand, the origin of the secondary electron contrast for atomic layers is not well understood, thus the control of the image contrast is not performed systematically. Nevertheless, in situ UHV-SEM has been proved useful for the observations of surface transitions, epitaxial growth processes and nano-fabrications.

Atomic step dynamics: The motion of atomic steps on $\operatorname{Si}(111)$ " $1 \times 1$ " is studied using ultra-flat $\mathrm{Si}(111)$ substrates that have flat terraces of $100 \mu \mathrm{m}$ in width [5]. Atomic steps are revealed by (7×7)-domain decoration. Step-flow growth and sublimation processes are compared for single- step-high islands and holes. The step motion is highly symmetric for growth in comparison to sublimation, and for islands in comparison to holes. The step motion fits well with the simple model by Barton, Cabrera and Frank [6]. No clear asymmetry in step kinetics is observed for upper terraces in comparison to lower terraces. However, by introducing an asymmetry in the relative terrace sizes on either side of a step, the adatom flux balance is tilted, and the result is a morphological instability in step flow (FIG. 1) [7].

Nanostructure arrangement: Ordered networks of atomic step bunches are created by annealing a Si substrate containing an array of small holes in UHV [8]. When the diffusion length of deposited materials is comparable to the step network size, only one island remains in every network unit. Au or Ga liquid islands accumulate at a particular position of the step bunch in each pattern unit during annealing (FIG. 2) [9]. This phenomenon is interpreted in terms of the energetic stability of a droplet on the patterned surface.

Combined microscopy: We have combined an STM unit or other micro probes for electric measurements with SEM. The SEM/STM system presents complementary images in atomic and macroscopic scales, which is useful for nanostructure fabrication. The micro probes in SEM are positioned to measure the electric properties of carbon nanotubes grown between electrodes (FIG. 3). The combination of SEM and nano-scale measurement/manipulation is a new way to go.

\section{References}

[1] Y. Homma, M. Tomita and T. Hayashi, Surf. Sci. 258 (1991) 147; Ultramicrosc. 52 (1993) 187.

[2] Y. Homma, M. Suzuki, M. Tomita, Appl. Phys. Lett. 62 (1993) 3276.

[3] Y. Homma, J. Osaka and N. Inoue, Surf. Sci. 357 (1996) 441.

[4] Y. Homma, P. Finnie and T. Ogino, Microbeam Analysis 2000, Institute of Physics Publishing, Bristol, 2000, pp 375-376.

[5] P. Finnie, Y. Homma, Phys. Rev. B 62 (2000) 8313.

[6] W. K. Burton, N. Cabrera, and F. C. Frank, Philos. Trans. R. Soc. London, 243 (1951) 299.

[7] Y. Homma, P. Finnie and M. Uwaha, Surf. Sci. 492 (2001) 125.

[8] T. Ogino, H. Hibino and Y. Homma, Appl. Surf. Sci. 117/118 (1977) 642.

[9] Y. Homma, P. Finnie and T. Ogino, Appl. Phys. Lett. 74 (1999) 815. 


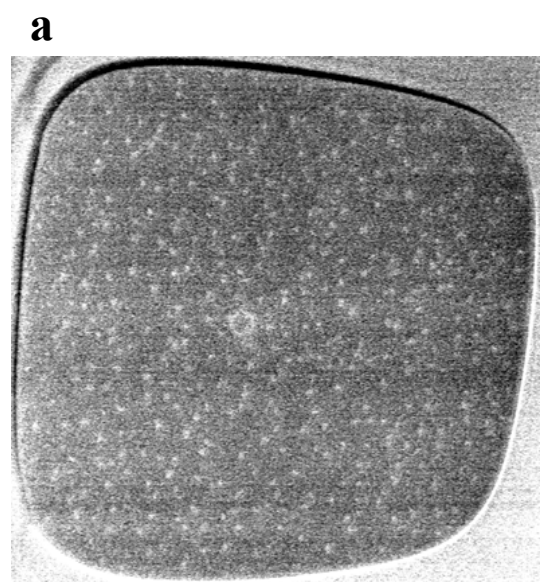

b

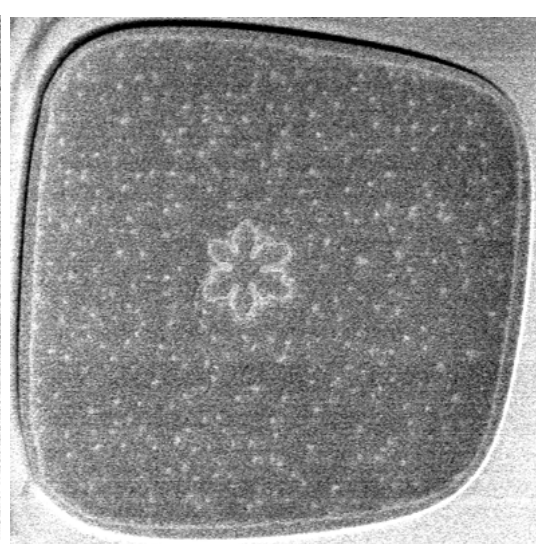

FIG. 1. SEM images of unstable step-flow observed during growth of $\mathrm{Si}(111)$ at $880^{\circ} \mathrm{C}$. Growth duration: (a) $20 \mathrm{~s}$; (b) $1 \mathrm{~min}$; (c) $5 \mathrm{~min}$. c

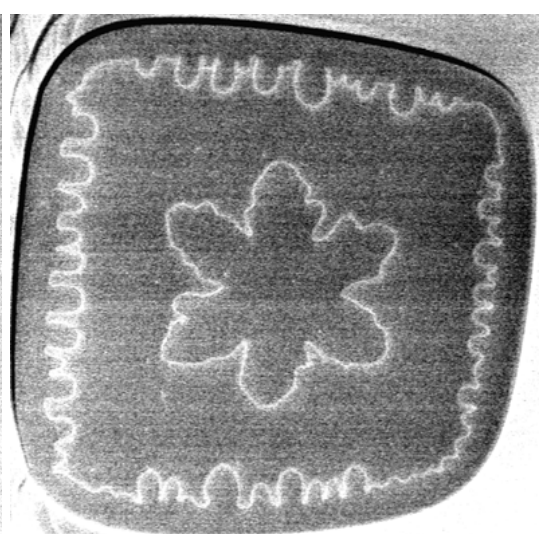

$50 \mu \mathrm{m}$
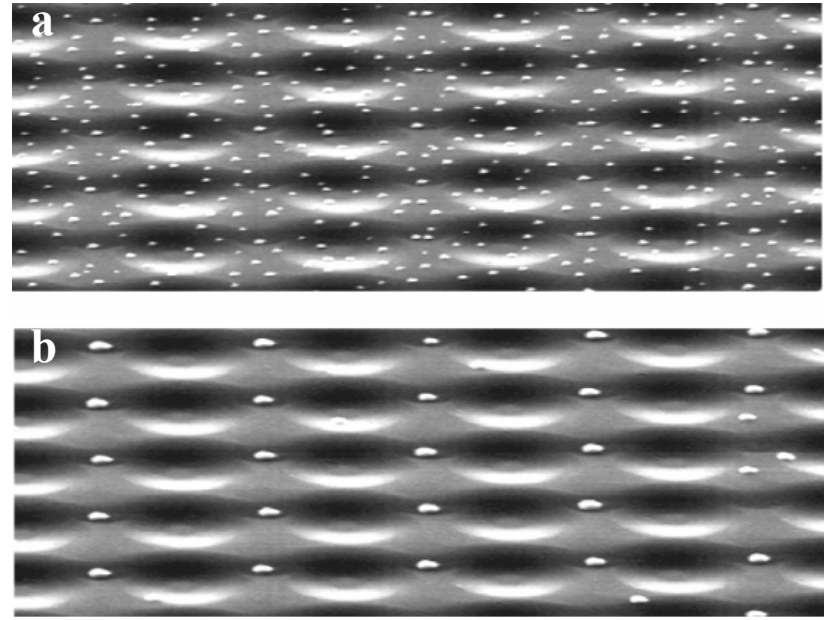

$\mathbf{3} \mu \mathbf{m}$

FIG. 2. SEM images of Au-island alignment on a step arranged substrate during annealing at $560^{\circ} \mathrm{C}$. Annealing duration: (a) $1 \mathrm{~min}$; (b) $26 \mathrm{~min}$.

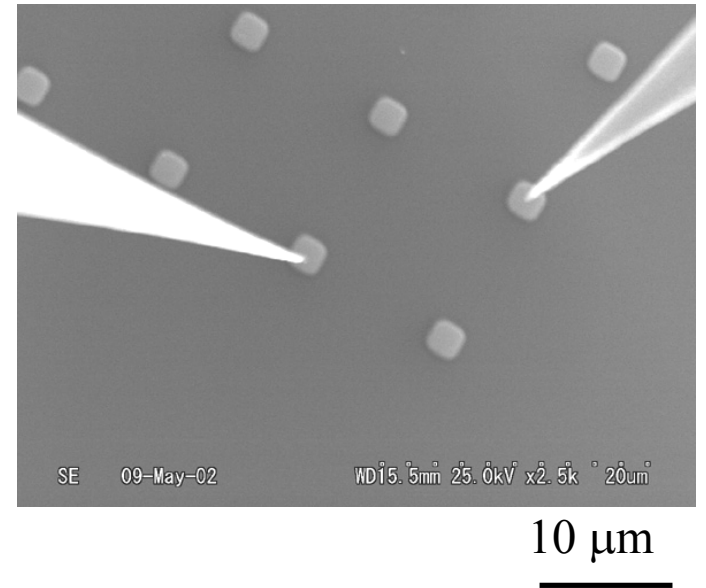

FIG. 3. SEM image of W-micro probes contacting with the electrodes observed in the combined microscope. 